\title{
Role of GLTSCR2 in the regulation of telomerase activity and chromosome stability
}

\author{
JEE-YOUN KIM, YONG-MIN AN and JAE-HOON PARK \\ Department of Pathology, School of Medicine, Kyung Hee University, Seoul 130-701, Republic of Korea
}

Received March 11, 2015; Accepted January 11, 2016

DOI: $10.3892 / \mathrm{mmr} .2016 .5427$

\begin{abstract}
Telomerase is essential for regulating telomeres, and its activation is a critical step in cellular immortalization and tumorigenesis. The transcriptional activation of human telomerase reverse transcriptase (hTERT) is critical for telomerase expression. Although several transcriptional activators have been identified, factors responsible for enhancing the hTERT promoter remain to be fully elucidated. In the present study, the role of glioma tumor-suppressor candidate region gene 2 (GLTSCR2) in telomerase regulation was analyzed. A doxycyclin-inducible green fluorescent protein (GFP)-tagged GLTSCR2-expressing adenovirus (Ad-GLT/GFP) was used for the transduction of SK-Hep-1 and T98G cancer cells, and normal human umbilical vein endothelial cells. Changes in telomerase activity using telomere repeat amplification protocol assay were assessed, and the gene expression levels of hTERT were then examined. To investigate chromosome instability and senescence, Giemsa and $\beta$-galactosidase staining was performed. The results revealed that overexpression of GLTSCR2 significantly increased telomerase activity in the cancer and normal cell lines. This increase was consistent with increases in the protein and mRNA expression levels of hTERT. In luciferase assays, the hTERT promoter was activated by GLTSCR2. Knockdown of GLTSCR2 led to the downregulation of telomerase activity, abnormal nuclear morphology as a marker of chromosome instability, significant suppression of growth rate, alterations in cellular morphology and, eventually, cellular senescence. Taken together, the results of the present study suggested that GLTSCR 2 is crucially involved in the positive regulation of telomerase and chromosome stability.
\end{abstract}

Correspondence to: Professor Jae-Hoon Park, Department of Pathology, School of Medicine, Kyung Hee University, 1 Hoegi-Dong Dongdaemun-Gu, Seoul 130-701, Republic of Korea

E-mail: jhpark@khu.ac.kr

Key words: glioma tumor-suppressor candidate region gene 2, telomerase activity, chromosome stability

\section{Introduction}

Telomerase is essential for maintaining telomeres, and consists of an RNA template and a catalytic protein subunit, telomerase reverse transcriptase (TERT), which adds a six-base DNA repeat sequence (TTAGGG) to the telomere (1). With decreasing telomerase activity, the ends of chromosomes become shortened as somatic cells age or mature $(2,3)$. Extensive efforts have been made to clarify the molecular mechanisms underlying telomerase activation. Human (h) TERT is a critical determinant of telomerase activation within the cell $(4,5)$. Although a number of transcription factors have been identified as regulators of the hTERT promoter (6), the specific factors responsible for determining telomerase activity in cells remain to be fully elucidated.

Previously, the nucleolar protein, GLTSCR2, was shown to be a putative tumor suppressor gene, as it is capable of inducing phosphatase and tensin homolog-dependent apoptotic cell death and inhibiting tumor growth (7-9). However, the biological function and molecular mechanisms of GLTSCR2 remain to be fully elucidated. In our previous study, it was reported that GLTSCR2 functions as a DNA damage response protein in the ataxia telangiectasia mutated (ATM)-Chk2 and ataxia telangiectasia and Rad3-related protein (ATR)-Chk1 pathways (10). Notably, increasing evidence indicates that several DNA damage response proteins are involved in telomere maintenance. Mutations in proteins involved in the response to DNA damage result in telomere dysfunction and subsequent chromosomal instability, suggesting extensive functional interactions between telomere maintenance and DNA damage response mechanisms (11-14). In the present study, the effect of GLTSCR2 on telomerase activity in various cell types was examined. Subsequent to this, the effect of the knockdown of GLTSCR2 on cellular morphology and growth rate was investigated. The present study revealed a novel biological function of GLTSCR2 in maintaining chromosomal stability via telomerase regulation.

\section{Materials and methods}

Cell culture and treatment. The SK-Hep-1 cells, T98G cells and human umbilical vein endothelial cells (HUVECs) were obtained from the American Type Culture Collection (Rockville, MD, USA). The cells were cultured in a humidified 
$5 \% \mathrm{CO}_{2}$ incubator maintained at $37^{\circ} \mathrm{C}$, either in Dulbecco's modified Eagle's medium (Invitrogen; Thermo Fisher Scientific, Inc., Waltham, MA, USA; for SK-Hep-1 and T98G cells) or in endothelial growth medium-2 (HUVECs; Thermo Fisher Scientific, Inc) supplemented with $10 \%$ fetal bovine serum (Thermo Fisher Scientific, Inc).

Antibodies and reagents. The anti-GLTSCR2 polyclonal antibody was produced by the present group by immunizing a rabbit with keyhole limpet hemocyanin-conjugated amino-acid residues 78-193 (CTRAKPGPQDTVERPF) of human GLTSCR2. The antibody was purified from the immune serum by affinity chromatography (8). The rabbit polyclonal anti-human TERT $(1: 1,000 ;$ SC-7212) and the rabbit polyclonal anti-tubulin antibodies (1:1,000; SC-9104) were purchased from Santa Cruz Biotechnology, Inc. (Santa Cruz, CA, USA). All other reagents were obtained from Sigma-Aldrich (St. Louis, MO, USA), unless otherwise specified.

Construction of the Tet-Off adenoviral-mediated system. A GFP-GLTSCR 2 construct consisting of full-length GLTSCR2 cDNA fused to a C-terminal GFP tag was first cloned into pTRE-Shuttle2 vector (Clontech Laboratories, Inc., Mountain View, CA, USA), which contains the tetracyclineresponsive element (TRE) upstream of the CMV minimal promoter. The resulting TRE-GFP-GLTSCR2 expression unit was excised from the pTRE-Shuttle2 vector using I-Ceu I and PI-Sce I resection enzymes, and then ligated to Swa I-digested Adeno-X System 1 Viral DNA (Clontech). The resulting recombinant Adeno-XGFP-GLTSCR2 vector (Ad-GFP/GLT) was packaged into infectious adenoviral particles by transfection of HEK293 cells, and recombinant adenovirus was harvested by lysing transfected cells. In the Tet-off system, the tet-responsive transcriptional activator is expressed and binds to the TRE in the absence of doxycycline (Clontech; stored in aliquots and kept at $-20^{\circ} \mathrm{C}$ ) to activate transcription of GLTSCR2. As doxycycline is added to the culture medium at $37^{\circ} \mathrm{C}$, transcription from the TRE is turned off in a dose dependent manner. To transiently express GLTSCR2, the cells were plated in a 6 -well plate at $70 \%$ confluence $\left(5 \times 10^{5}\right.$ cells $)$ and coinfected with a recombinant adenovirus, [Ad-green fluorescent protein (GFP)/GLT], and a regulation virus (Adeno-X Tet-Off virus) with a multiplicity of infection (MOI) of 100 in serum-free media for $12 \mathrm{~h}$. Fresh complete medium was subsequently added.

Generation of stable GLTSCR2-knockdown cell lines. For knockdown of the expression of GLTSCR2, feline leukemia virus-based lentiviral GLTSCR 2 shRNA vectors were purchased from GeneCopoeia, Inc. (Rockville, MD, USA). The GLTSCR2-target sequence was 5'-GAGACCGGTTCA AGAGCTT-3', and the scrambled (Scr) sequence was 5'-CGA TACTGAACGAATC-3'. Cells were seeded at $5 \times 10^{5}$ cells in $30 \mathrm{~mm}$ dishes and lentiviral stocks of the GLTSCR 2 shRNA or the control shRNA vector were then incubated with separate sets of cells with an MOI of 10 . After $48 \mathrm{~h}$, clones of GLTSCR2-knockdown cells were selected for using puromycin ( $1 \mu \mathrm{g} / \mathrm{ml}$; Sigma-Aldrich) treatment. Protein expression levels were analyzed using western blot and immunocytochemical analyses.
Telomere repeatamplification protocol(TRAP). A TRAP assay was used to detect telomerase activity, in which a TRAPEZE ${ }^{\circledR}$ XL telomerase detection kit (EMD Millipore, Billerica, MA, USA) was used, according to the manufacturer's protocol. Briefly, cell pellets were resuspended in $200 \mu 1$ of 3 -[chola midoprophyl)-dimethylammonio]-1-propane-sulfonate lysis buffer and incubated for $30 \mathrm{~min}$ on ice. After centrifugation at $13,000 \mathrm{~g}$ for $20 \mathrm{~min}$ at $4^{\circ} \mathrm{C}$, aliquots of the supernatant were rapidly frozen and store $-80^{\circ} \mathrm{C}(15)$. The telomeric DNA from the cell extracts was amplified by 36 cycles of polymerase chain reaction (PCR) with a denaturation at $94^{\circ} \mathrm{C}$ for $30 \mathrm{sec}$, annealing at $59^{\circ} \mathrm{C}$ for $30 \mathrm{sec}$, and elongation at $72^{\circ} \mathrm{C}$ for $1 \mathrm{~min}$. $20 \mu \mathrm{l}$ of the PCR product were subjected to electrophoresis on a non-denaturing $10 \%$ polyacrylamide gel (Sigma-Aldrich) The experiment was repeated four times.

Reverse transcription semi-quantitative PCR (RT-PCR) analysis. Total RNA (1 $\mu \mathrm{g})$ was extracted using Trizol reagent (Invitrogen) from the cultured cells and converted into cDNA using a cDNA synthesis kit (Takara Bio, Inc., Otsu, Japan), according to the manufacturer's protocol. hTERT and tubulin mRNA amplification were performed with the following primers: hTERT forward, 5'-AGAGTGTCTGGAGCAAGTTGC-3', hTERT reverse, 5'-CGTAGTCCATGTTCACAATCG-3'; tubulin forward, 5'-CATGTATCTTCCATACCCTG-3', tubulin reverse, 5'-CTGAAGGTATTCATGATGCG-3'. The cycling conditions were as follows: Denaturation at $94^{\circ} \mathrm{C}$ for $30 \mathrm{sec}$, annealing at $55^{\circ} \mathrm{C}$ for $30 \mathrm{sec}$, elongation at $72^{\circ} \mathrm{C}$ for $30 \mathrm{sec}$. The resulting PCR products were separated on $2 \%$ agarose gels (Sigma-Aldrich) and visualized using ethidium bromide staining $(0.5 \mu \mathrm{g} / \mathrm{ml}$; Sigma-Aldrich). The quantification of the transcriptional gene expression was performed using the Gel Doc EZ system Image Lab ${ }^{\mathrm{TM}}$ software (Bio-Rad Laboratories, Inc., Richmond, CA, USA) and normalized to the expression of tubulin as an endogeneous control. The method used for quantification was semi-quantitative evaluation using densitometric analysis (16)

Luciferase assay. The pGL2 luciferase plasmid (Promega Corporation, Madison, WI, USA) was used. The hTERT promoter sequence was amplified by PCR to generate a $1580 \mathrm{bp}$ fragment from human genomic DNA using upstream primers (5'-AGCGATACCTATTGAATGCC-3') containing a $K p n I$ site, together with a single downstream primer (5'-TCTCCTCGCGGCGCGAGTTT-3') containing a HindIII site. Luciferase activity was measured in samples containing equivalent quantities of protein using a luminometer and luciferase assay reagents (Promega Corporation). The protein content of cell lysates was determined using BCA protein assay reagent (Pierce Biotechnology, Inc., Rockford, IL, USA) in clear 96-well plates (Nunc, Wiesbaden, Germany), and colorimetric assay was performed using an VersaMax ELISA Microplate reader (Molecular Devices, LLC, Sunnyvale, CA, USA). The equivalent quantities of protein were used in the subsequent luciferase activity assay.

Giemsa staining and slide preparation. Fixed slides were immersed in 5\% Giemsa (Sigma-Aldrich) for $20 \mathrm{~min}$. The slides were then rinsed briefly in $\mathrm{dH}_{2} \mathrm{O}$ and air-dried. For 
A
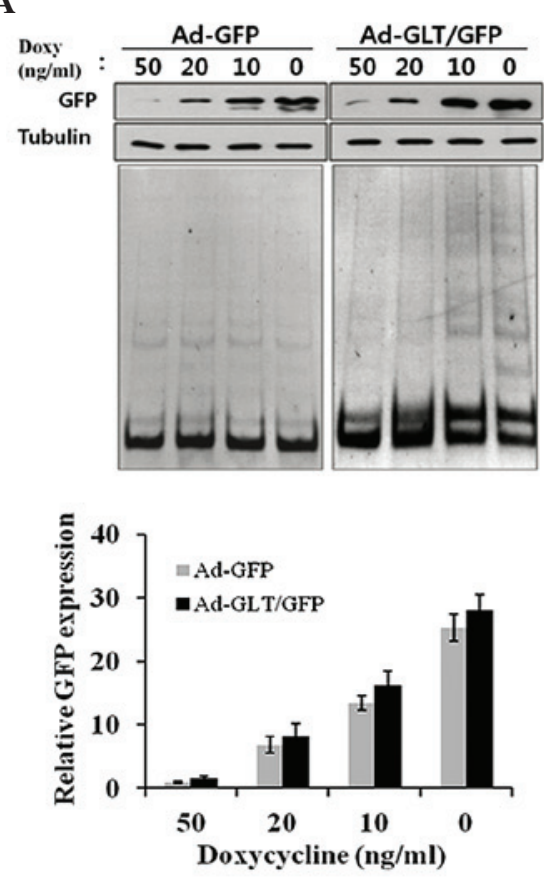

B
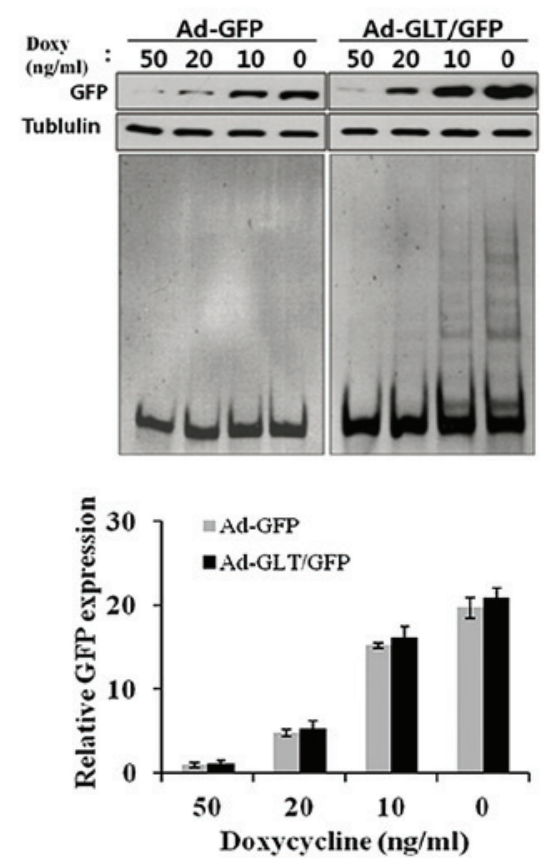

C
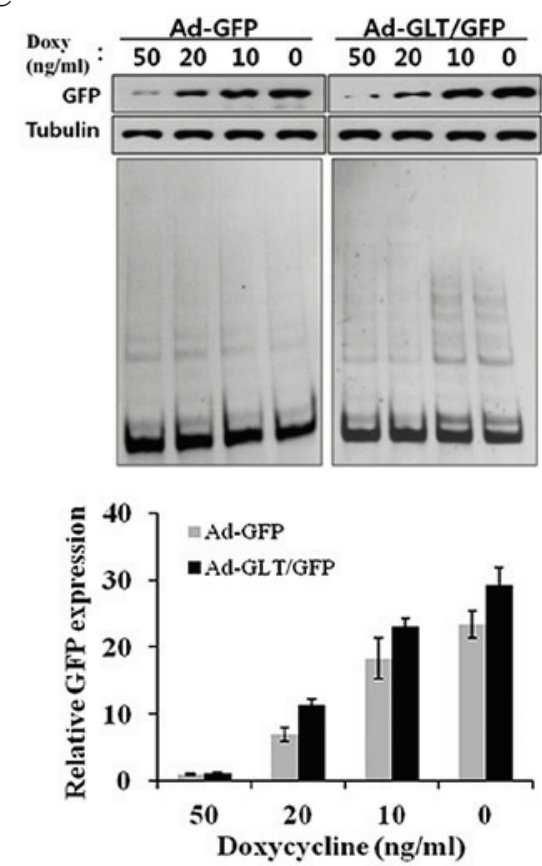

D
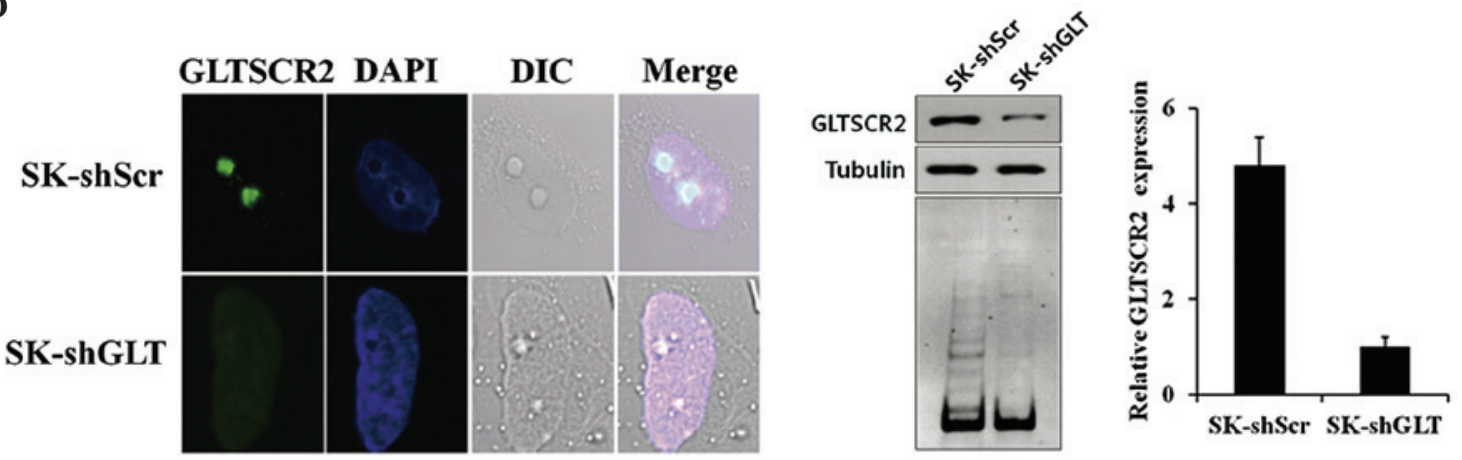

Figure 1. GLTSCR2 increases telomerase activity. (A) SK-Hep-1 cells, (B) T98 G cells and (C) human umbilical vein endothelial cells were infected with Ad-GFP or Ad-GLT/GFP, and incubated with decreasing concentrations of doxycycline (50, 20, $10 \mathrm{and} 0 \mathrm{ng} / \mathrm{ml}$ ). (D) SK-Hep-1 cells were stably infected with lentivirus carrying either GLTSCR2-targeting SK-shGLT or SK-shScr, immunostained with anti-GLTSCR2 and DAPI, and viewed under an DIC-equipped confocal microscope (Left, magnification x630). Cells were harvested, and lysates were examined using western blotting with the indicated antibodies. Tubulin was used as a loading control. TRAP assays were performed using subconfluent proliferating cultures of cells. GLT, glioma tumor-supressor; GLTSCR2, glioma tumor-suppressor candidate region gene 2; GFP, green fluorescent protein; sh, short hairpin RNA; Scr, scambled; Doxy, doxycycline.

long-term storage, the slides were mounted with a drop of Permount (Thermo Fisher Scientific, Inc.) and coverslips.

Senescence-associated $\beta$-galactosidase staining. The cells were fixed with $2 \%$ formaldehyde $/ 0.2 \%$ glutaraldehyde solution (both Cell Signaling Technology, Inc., Danvers, MA, USA) and stained overnight at $37^{\circ} \mathrm{C}$ with $1 \mathrm{mg} / \mathrm{ml} \mathrm{X-gal}$ (Cell Signaling Technology, Inc.), $40 \mathrm{mM}$ citric acid $/ \mathrm{Na}_{2} \mathrm{HPO}_{4}$ (pH 6.0; Sigma-Aldrich), $5 \mathrm{mM}$ potassium ferrocyanide/ferricyanide (Cell Signaling Technology, Inc.), $150 \mathrm{mM} \mathrm{NaCl}$ and $2 \mathrm{mM} \mathrm{MgCl}$, both Sigma-Aldrich.

Statistical analysis. In all cases, the results are presented as the mean \pm standard deviation. Statistical analysis was performed using SPSS software, version 12.0 (SPSS, Inc., Chicago, IL, USA). A two-tailed, unpaired Student's $t$-test was used for data analysis. $\mathrm{P}<0.05$ was considered to indicate a statistically significant difference.

\section{Results}

To determine the effect of GLTSCR2 on telomerase activity, the present study first measured the level of telomerase activity in the cultured SK-Hep-1 cells. Initially, the SK-Hep-1 cells were infected with a doxycycline-inducible (Tet-Off system) adenovirus, expressing either GFP-tagged GLTSCR2 (Ad-GLT/GFP) or GFP (Ad-GFP) as a control, and placed in media containing different concentrations of doxycycline $(0,10,20$ and $50 \mathrm{ng} / \mathrm{ml})$ for $48 \mathrm{~h}$. Telomerase activity was measured using a TRAP assay, as described above. As shown in Fig. 1A, the Ad-GLT/GFP cells showed significantly increased telomerase activity in proportion to the expression levels of GLTSCR2, compared with the Ad-GFP cells. The effects of GLTSCR2 in the T98G glioblastoma cells were similar to those observed in the SK-Hep-1 cells (Fig. 1B).

The majority of cancer cells express high levels of telomerase activity. However, human primary normal cells usually 
A

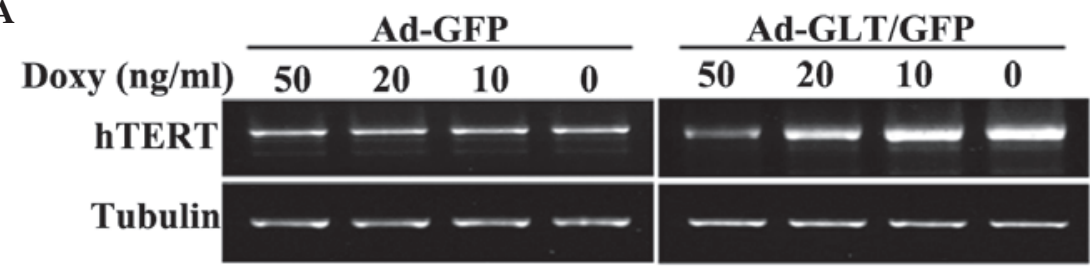

B

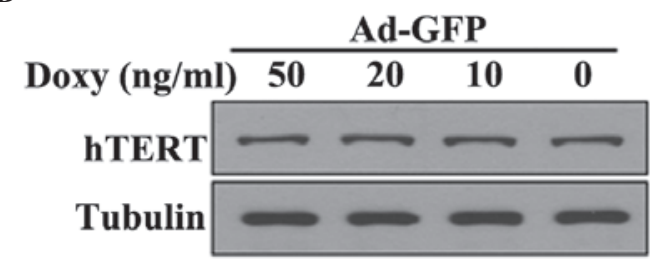

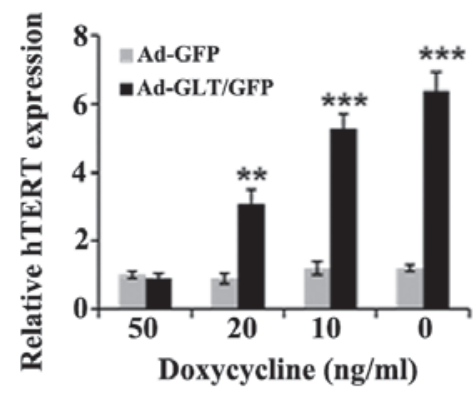

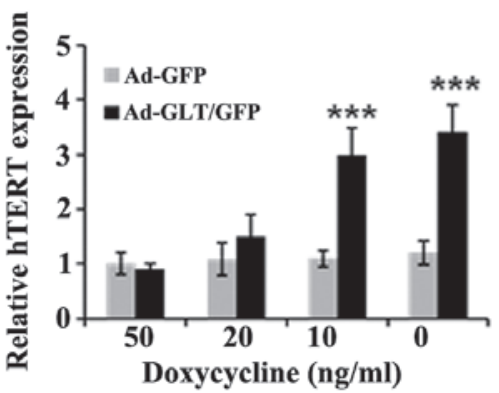

Figure 2. Overexpression of GLTSCR2 causes upregulation of the mRNA and protein expression of hTERT. SK-Hep-1 cells were infected by Ad-GFP or Ad-GLT/GFP. The cells were then harvested to isolate RNA for (A) semi-quantitative RT-PCR assays or to prepare whole-cell lysates for (B) western blotting The expression level of hTERT in the GLTSCR2-overexpressing Ad-GFP/GLT cells was significantly higher than those in the corresponding control (Ad-GFP) cells. Expression levels were quantified by densitometric analysis of banding intensity. Data are presented as the mean \pm standard deviation of three independent experiments. ${ }^{* *} \mathrm{P}<0.01,{ }^{* * *} \mathrm{P}<0.001$ vs. Doxy $50 \mathrm{ng} / \mathrm{ml}$. hTERT, human telomerase reverse transcriptase; GLT, glioma tumor-supressor; GLTSCR2, glioma tumor-suppressor candidate region gene 2; GFP, green fluorescent protein; Doxy, doxycycline.

A

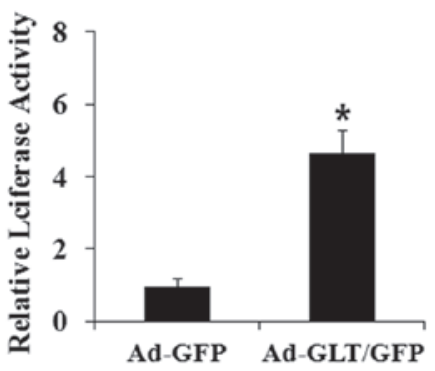

B

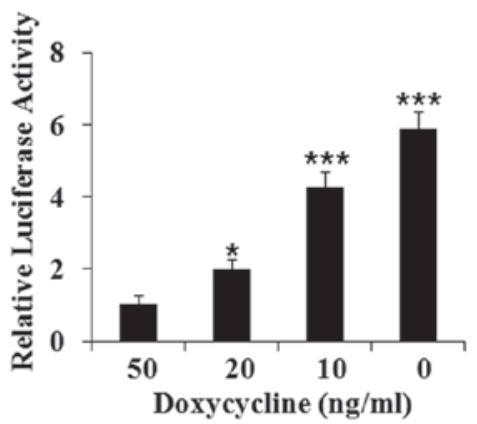

Figure 3. GLTSCR2 increases the transcriptional activity of the hTERT promoter. (A) SK-Hep-1 cells were transfected with the hTERT promoter plasmid using Lipofectamine for $24 \mathrm{~h}$, and then infected with Ad-GFP or Ad-GLT/GFP. Following another $24 \mathrm{~h}$ incubation, the cell lysates were extracted, and luciferase assays were performed. hTERT promoter activity was increased in the GLTSCR2-overexpressing cells ${ }^{* * *} \mathrm{P}<0.001$ vs. Ad-GFP. (B) SK-Hep-1 cells were infected with Ad-GFP or Ad-GLT/GFP with decreasing concentrations of doxycycline (50, 20, $10 \mathrm{and} 0 \mathrm{ng} / \mathrm{ml}$, respectively). hTERT promoter activity was induced dose-dependently by GLTSCR2. Data are presented as the mean \pm standard deviation of three independent experiments. ${ }^{*} \mathrm{P}<0.05,{ }^{* * * *} \mathrm{P}<0.001 \mathrm{vs}$. Doxy $50 \mathrm{ng} / \mathrm{ml}$. hTERT, human telomerase reverse transcriptase; Doxy, doxycycline.

exhibit a low level of telomerase activity $(17,18)$. If GLTSCR2 is an inducer of telomerase activity, its expression may induce telomerase activity in primary normal cells. Thus, the present study examined the effect of GLTSCR on telomerase activity in HUVECs. The HUVECs were infected with Ad-GLT/GFP or Ad-GFP for $48 \mathrm{~h}$, and the TRAP assay was performed. Telomerase activity was low and almost undetectable in the Ad-GFP HUVEC cells. However, telomerase activity in the Ad-GLT/GFP HUVEC cells was significantly increased and correlated with the expression level of GLTSCR2, which was similar to the results observed in the SK-Hep-1 and T98G cells (Fig. 1C).

To further demonstrate the effect of GLTSCR2 on telomerase activity, the SK-Hep-1 cells were stably infected with a lentivirus carrying either GLTSCR2-targeting shGLT or shScr. Subsequent immunofluoresence and immunoblotting confirmed that the expression of GLTSCR 2 was significantly reduced, by $>80 \%$, in the cells stably infected with shGLT (Fig. 1D). In contrast to the GLTSCR2-overexpressing SK-Hep-1 cells, the SK-shGLT cells showed lower telomerase activity, compared with the control (SK-shScr) cells (Fig. 1D). These results suggested that GLTSCR2 exerted a positive effect on telomerase activity.

To investigate the mechanism by which GLTSCR2 increases telomerase activity, the present study examined whether GLTSCR2 affected the expression of hTERT, a catalytic subunit of telomerase and a critical determinant of telomerase activity. The SK-Hep-1 cells were infected with 
A
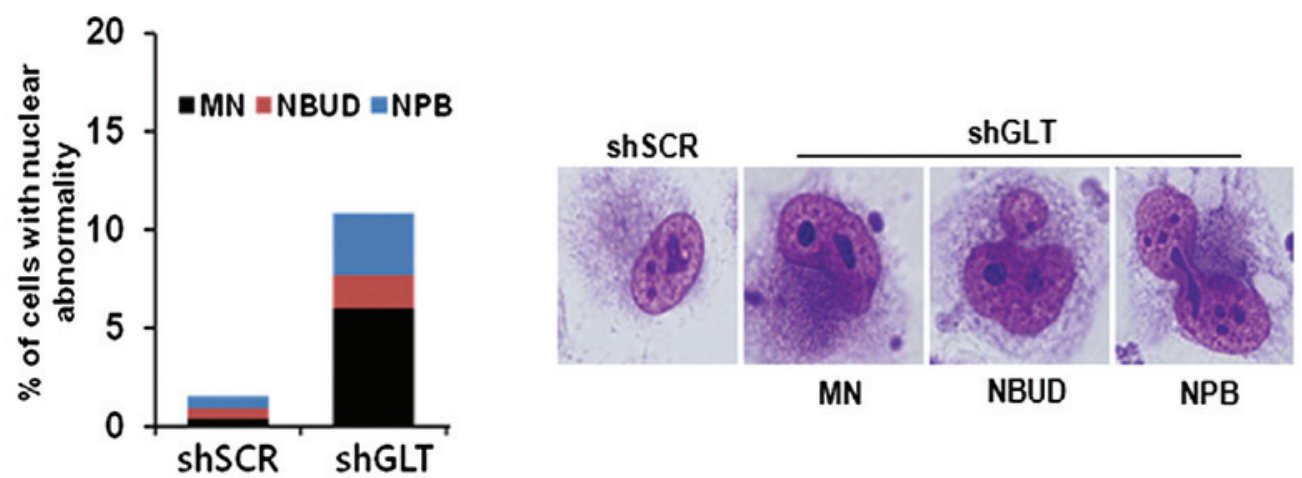

B

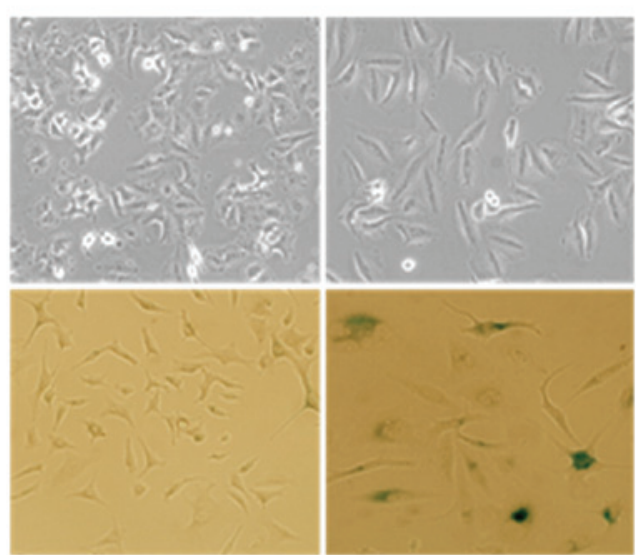

C

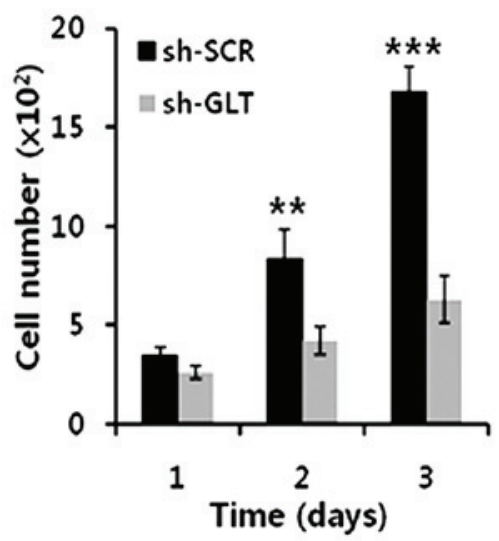

Figure 4. Knockdown of GLTSCR2 induces abnormal nuclear morphology and cellular senescence. The T98G cells were stably infected with a lentivirus carrying either GLTSCR2-targeting shGLT or scrambled shRNA. (A) T98G-shGLT cells showed nuclear abnormalities, visualized by staining with Giemsa, including MN, NBUD and NPB, magnification x630. A minimum of 300 cells were counted and the count was performed three times. (B) Top and bottom panels show morphological changes and cellular senescence by $\beta$-galatosidase staining, in the T98G-shScr and T98G-shGLT groups. Magnification, x100. (C) Growth rates of the cells were determined by total cell count at the time points indicated. Data are presented as the mean \pm standard deviation of three independent experiment. sh, short hairpin RNA; GLT, glioma tumor-supressor; GLTSCR2, glioma tumor-suppressor candidate region gene 2; Scr, scambled; MN, micronucleus; NBUD, nuclear buds; NPB. nucleoplasmic bridges. ${ }^{* *} \mathrm{P}<0.01,{ }^{* * *} \mathrm{P}<0.001$ vs. shSCR 1 day.

Ad-GLT/GFP or Ad-GFP for $48 \mathrm{~h}$. The mRNA and protein expression levels of hTERT were then evaluated using RT-PCR analysis and western blotting, respectively. Compared with the Ad-GFP control cells, the Ad-GLT/GFP cells exhibited increased mRNA and protein levels of hTERT (Fig. 2A and B).

To investigate the mechanism underlying the GLTSCR2-induced expression of hTERT, the present study examined the effect of GLTSCR 2 on the activity of the hTERT gene promoter by using the hTERT promoter-luciferase reporter plasmid, pGL2-hTERT, in the SK-Hep-1 cells. The SK-Hep-1 cells were transiently transfected for $24 \mathrm{~h}$ with pGL2-hTERT, and were then infected with either Ad-GLT/GFP or Ad-GFP for $24 \mathrm{~h}$ to allow for the expression of GLTSCR2. As shown in Fig. 3A, Ad-GLT/GFP markedly induced hTERT promoter activity, whereas Ad-GFP had minimal or no effect on hTERT promoter activity. The induction of pGL2-hTERT activity by GLTSCR2 was dose-dependent (Fig. 3B). Together, these data demonstrated consistent positive regulation of the hTERT gene by GLTSCR2 in the SK-Hep-1 cells.

Telomeres are essential for maintaining chromosome stability, and extensive telomere shortening can lead to abnormal nuclear morphologies, including micronuclei, nucleoplasmic bridges or nuclear buds as a marker of chromosome instability (19). Subsequently, the effect of the knockdown of
GLTSCR2 on abnormal nuclear morphology was examined. The presence of micronuclei, nucleoplasmic bridges and nuclear buds increased in the T98G-shGLT cells (Fig. 4A).

The present study also examined the effects of the knockdown of GLTSCR2 on cellular morphology and growth rate. The T98G-shGLT cells presented with significant morphological changes, characterized by an elongated and enlarged flat shape, resembling the morphology of senescent cells, compared with parental T98 G cells (Fig. 4B). The growth rate was reduced in the T98G-shGLT cells (Fig. 4C). Additionally, the T98G-shGLT cells entered cellular senescence, as determined by senescence-associated $\beta$-galactosidase staining (Fig. 4B). Taken together, these results suggested that GLTSCR 2 is crucially involved in the positive regulation of telomerase and chromosome stability.

\section{Discussion}

In the present study, to investigate the effect of GLTSCR2 on telomerase activity, human SK-Hep-1 and T98G cancer cells, and normal HUVECs were infected with Ad-GLT/GFP in a doxycycline-inducible (Tet-Off system) manner. Subsequently, changes in telomerase activity and the expression of hTERT were determined.GLTSCR2 significantly increased telomerase 
activity in the cancer and normal cells. This increase was consistent with an increase in the expression pf hTERT, as determined by semi-quantitative RT-PCR analysis, western blot analysis and promoter assays for hTERT. Although the molecular mechanism underlying the regulation of telomerase activity by GLTSCR 2 remains to be fully elucidated and currently under investigation, these results suggested that GLTSCR2 is crucially involved in the positive regulation of telomerase maintenance.

Several other studies have been performed to identify the transcriptional factors critical for hTERT regulation (20-22), however, no specific factors have been identified, which are responsible for determining telomerase activity in cells. It is now widely accepted that a number of nuclear factors are involved in the regulation of hTERT transcription $(23,24)$. Furthermore, hTERT undergoes a regulated subnuclear translocation between the nucleoplasm and the nucleolus (25). hTERT nucleolar transportation may be an essential step for the biogenesis of telomerase in human cells $(26,27)$. The telomere-capping and stabilizing proteins, telomeric repeat binding factor (TRF)1 and TRF2, also accumulate in the nucleolus, and they are modulated by nucleolar proteins (28). Within these complex regulatory mechanisms, GLTSCR2 appears to be involved. The present study suggested that the GLTSCR2 nucleolar protein is a crucial determinant of telomerase activity in normal and cancer cells.

Human primary cells usually exhibit low levels of telomerase activity. However, the majority of cancer cell lines express high levels of telomerase activity, which may support continued cell proliferation $(17,18)$. In GLTSCR2-overexpressing SK-Hep-1 cells and control cells, no significant differences in cell proliferation were observed (data not shown), although telomerase activity was significantly increased in the former. By contrast, in the GLTSCR2-knockdown cells, cell proliferation was significantly lower, compared with that in the control cells. These results indicated that it is unlikely that cell proliferation contributed to the increased telomerase activity observed in the GLTSCR2-overexpressing cell lines. However, in the GLTSCR2-knockdown cells, decreased telomerase activity may have impeded proliferation. Thus, GLTSCR2, but not the rate of cell proliferation, appears to be important in telomerase activity.

Reports have indicated that the cellular DNA damage response (DDR) ensures genomic stability and protects against genotoxic stresses. By contrast, defects in the DDR contribute to genomic instability (10). For example, defects in ATM, a key DNA damage signaling molecule, are associated with telomere loss, telomeric fusions and extrachromosomal telomeric DNA appearance in cells from patients with ataxia telangiectasia and ATM-deficient mice (12). In addition, the TRF1 and TRF2 proteins, which stabilize telomeres, are known to be important in the DDR. In particular, the inhibition of the DDR by dominant negative alleles or by the genetic deletion of TRF2 in mouse cells gives rise to a potent checkpoint response and frequent end-to-end fusions (29). Furthermore, TRF2 is rapidly phosphorylated in response to DNA damage, likely via an ATM-kinase-mediated pathway, which is critical for telomere maintenance $(12,30)$. Upon phosphorylation, TRF2 rapidly localizes to sites of DNA damage, specifically double-strand breaks, where it acts as an early component of the DNA repair response system (14). GLTSCR2 mediates the activation of ATM-Chk2 and ATR-Chk1 in response to DNA damage (10). In the present study, it was shown that the siRNA-based knockdown of GLTSCR2 led to abnormal nuclear morphology, including the presence of micronuclei, nucleoplasmic bridges and nuclear buds as a indicator of chromosome instability, which indicated an impaired response of the cells to DNA damage.

In conclusion, the results of the present study suggested that GLTSCR2 is a crucially involved in the positive regulation of telomerase and chromosome stability. The precise role of GLTSCR2 in the regulation of telomerase remains to be elucidated. However, GLTSCR2-induced telomerase regulation may provide important clues regarding the role of GLTSCR2 in chromosome stability. Continued investigations into other GLTSCR2-associated proteins involved in the DDR are critical to determine the extent of overlap between telomere maintenance and DNA repair response signaling pathways.

\section{Acknowledgements}

The present study was supported by grants from the National Research Foundation of Korea funded by the Korean government (Ministry of Science, ICT and Future Planning; grant nos. 2011-0030072 and NRF-2013R1A2A2A01009006).

\section{References}

1. Oh BK, Yoon SM, Lee CH and Park YN: Rat homolog of PinX1 is a nucleolar protein involved in the regulation of telomere length. Gene 400: 35-34, 2007.

2. Bekaert S, Derradji H and Baatout S: Telomere biology in mammalian germ cells and during development. Dev Biol 274: 15-30, 2004.

3. Wills LP, Schnellmann RG: Telomeres and telomerase in renal health. J Am Soc Nephrol 22: 39-41, 2011.

4. Nakamura TM, Morin GB, Chapman KB, Weinrich SL, Andrews WH, Linger J, Harley CB and Cech TR: Telomerase catalytic subunit homologs from fission yeast and human. Science 277: 955-959, 1997.

5. Meyerson M, Counter CM, Eaton EN, Ellisen LW, Steiner P, Caddle SD, Ziaugra L, Beijersbergen RL, Davidoff MJ, Liu Q, et al: hEST2, the putative human telomerase catalytic subunit gene, is up-regulated in tumor cells and during immortalization. Cell 90: 785-797, 1997.

6. Hashimoto M, Kyo S, Hua X, Tahara H, Nakajima M, Takakura M, Sakaguchi J, Maida Y, Nakamura M, Ikoma T, et al: Role of menin in the regulation of telomerase activity in normal and cancer cells. Int J Oncol 33: 330-340, 2008.

7. Okahara F, Itoh K, Nakagawara A, Murakami M, Kanaho Y and Maehama T: Critical role of PICT-1, a tumor suppressor candidate, in phosphatidylinositol 3,4,5-trisphosphate signals and tumorigenic transformation. Mol Biol Cell 17: 4888-4895, 2006.

8. Yim JH, Kim YJ, Ko JH, Cho YE, Kim SM, Kim JY, Lee S and Park JH: The putative tumor suppressor gene GLTSCR2 induces PTEN-modulated cell death. Cell Death Differ 14: 1872-1879, 2007.

9. Okahara F, Ikawa H, Kanaho Y and Maehama T: Regulation of PTEN phosphorylation and stability by a tumor suppressor candidate protein. J Biol Chem 279: 45300-45303, 2004.

10. Kim JY, Seok KO, Kim YJ, Bae WK, Lee S and Park JH: Involvement of GLTSCR2 in the DNA damage response. Am J Pathol 179: 1257-1264, 2011.

11. Metcalfe JA, Parkhill J, Campbell L, Stacey M, Biggs P, Byrd PJ and Taylor AM: Accelerated telomere shortening in ataxia telangiectasia. Nat Genet 13: 350-353, 1996.

12. Hande MP, Balajee AS, Tchirkov A, Wynshaw-Boris A and Lansdorp PM: Extra-chromosomal telomeric DNA in cells from $\operatorname{Atm}(-/-)$ mice and patients with ataxia-telangiectasia. Hum Mol Genet 10: 519-528, 2001. 
13. Kishi S, Zho XZ, Ziv Y, Khoo C, Hill DE, Shiloh Y and Lu LP: Telomeric protein Pin2/TRF1 as an important ATM target in response to double strand DNA breaks. J Biol Chem 276: 29282-29291, 2001.

14. Bradshaw PS, Stavropoulos DJ and Meyn MS: Human telomeric protein TRF2 associates with genomic double-strand breaks as an early response to DNA damage. Nat Genet 37: 193-197, 2005.

15. Banerjee PP and Jagadeesh S: Non-radioactive assay methods for the assessment of telomerase activity and telomere length. Methods Mol Biol 1288: 305-316, 2015.

16. Marone M, Mozzetti S, De Ritis D, Pierelli L and Scambia G: Semiquentitative RT-PCR analysis to assess the expression levels of multiple transcripts from the same sample. Biol Proced Online 16: 19-25, 2001.

17. Hahn WC, Stewart SA, Brooks MW, York SG, Eaton E, Kurachi A, Beijersbergen RL, Kmoll JH, Meyerson M and Weinberg RA: Inhibition of telomerase limits the growth of human cancer cells. Nat Med 5: 1164-1170, 1999.

18. Kim NW, Piatyszek MA, Prowse KR, Harley CB, West MD Ho PL, Coviello GM, Wright WE, Weinrich SL and Shay JW: Specific association of human telomerase activity with immortal cells and cancer. Science 266: 2011-2015, 1994.

19. Gisselsson D, Björk J, Höglund M, Mertens F, Dal Cin P Akerman $\mathrm{M}$ and Mandahl N: Abnormal nuclear shape in solid tumors reflects mitotic instability. Am J Pathol 158: 199-206, 2011.

20. Horikawa I and Barrett JC: Transcriptional regulation of the telomerase hTERT gene as a target for cellular and viral oncogenic mechanisms. Carcinogenesis 24: 1167-1176, 2003.
21. Zhao Y, Cheng D, Wang S and Zhu J: Dual roles of c-Myc in the regulation of hTERT gene. Nucleic Acids Res 42: 10385-10398, 2014.

22. Daniel M, Peek GW and Tollefsbol TO: Regulation of the human catalytic subunit of telomerase (hTERT). Gene 498: 135-146, 2012

23. Chebel A, Rouault JP, Urbanowicz I, Baseggio L, Chien WW, Salles G and Ffrench M: Transcriptional activation of hTERT, the human telomerase reverse transcriptase, by nuclear factor of activated T cells. J Biol Chem 284: 35725-35734, 2009.

24. Gladych M, Wojtyla A and Rubis B: Human telomerase expression regulation. Biochem Cell Biol 89: 359-376, 2011.

25. Wong JM, Kusdra L and Collins K: Subnuclear shuttling of human telomerase induced by transformation and DNA damage. Nat Cell Biol 4: 731-736, 2002.

26. Yang Y, Chen Y,Zhang C, Huang H and Weissman SM: Nucleolar localization of hTERT protein is associated with telomerase function. Exp Cell Res 277: 201-209, 2002.

27. Mitchell JR, Cheng K and Collins K: A box H/ACA small nucleolar RNA-lie domain at the human telomerase RNA 3'end. Mol Cell Biol 19: 567-576, 1999.

28. Lin J, Jin R, Zhang B, Yang PX, Chen H, Bai YX, Xie Y, Huang $\mathrm{C}$ and Huang J: Characterization of a nobel effect of hPinX1 on hTERT nucleolar localization. Biochem Biophys Res Commum 353: 946-952, 2007.

29. Denchi EL: Give me a break: How telomeres suppress the DNA damage response. DNA Repair (Amst) 8: 1118-1126, 2009.

30. Tanaka H, Mendonca MS, Bradshaw PS, Hoelz DJ, Malkas LH, Meyn MS and Gilley D: DNA damage-induced phosphorylation of the human telomerase-associated protein TRF2. Proc Natl Acad Sci USA 102: 15539-15544, 2005. 